\title{
A HAMILTONIAN APPROACH TO FAULT ISOLATION IN A PLANAR VERTICAL TAKE-OFF AND LANDING AIRCRAFT MODEL
}

\author{
LUIS H. RODRIGUEZ-ALFARO ${ }^{a}$, EFRAIN ALCORTA-GARCIA $^{a, *}$, DAVID LARA $^{b}$, \\ GERARDO ROMERO ${ }^{b}$
}

\author{
${ }^{a}$ Faculty of Electrical and Mechanical Engineering \\ Autonomous University of Nuevo Leon, Av. Universidad s/n, San Nicolas de los Garza, N.L., Mexico c.p. 66451, Mexico \\ e-mail: efrain.alcortagr@uanl.edu.mx \\ ${ }^{b}$ Electrical Engineering Graduate School, UAM Reynosa Rodhe \\ Autonomous University of Tamaulipas, Reynosa Tamaulipas, Mexico
}

\begin{abstract}
The problem of fault detection and isolation in a class of nonlinear systems having a Hamiltonian representation is considered. In particular, a model of a planar vertical take-off and landing aircraft with sensor and actuator faults is studied. A Hamiltonian representation is derived from an Euler-Lagrange representation of the system model considered. In this form, nonlinear decoupling is applied in order to obtain subsystems with (as much as possible) specific fault sensitivity properties. The resulting decoupled subsystem is represented as a Hamiltonian system and observer-based residual generators are designed. The results are presented through simulations to show the effectiveness of the proposed approach.
\end{abstract}

Keywords: Hamiltonian, observer, fault diagnosis, nonlinear systems, UAVs.

\section{Introduction}

Aerial vehicles become more and more relevant because of many applications (Castillo et al., 2005). As any other system, aerial vehicles are also susceptible to the effect of faults. Reliability requirements in aerial vehicles motivate the necessity of a fault detection and isolation schema. In general, they are nonlinear systems, and so a nonlinear inspired strategy for the detection and isolation of faults could also be used (see, for example, Alcorta-Garcia and Frank, 1997; Krokavec and Filasová, 2012; Kościelny and Łabęda-Grudziak, 2013; Bartyś, 2013).

A model of an aerial vehicle that is able to take off vertically such as helicopters and some special airplanes is represented by the planar vertical take-off and landing (PVTOL) aircraft model. The PVTOL aircraft represents a challenging nonlinear system for control systems designers. This system represents a particular case of what is today known as "motion control".

In spite of many control strategies proposed in the literature (see, e.g., Fantoni et al., 2002; Al-Hiddabi et al., 1999; Lin et al., 1999; Castillo et al., 2002; Zavala

\footnotetext{
*Corresponding author
}

et al., 2003; Do et al., 2003; Wood and Cazzolato, 2007; Rejon and Aranda-Bricaire, 2006; Frye et al., 2010; Ailon, 2010), there are a restricted number of results related to the detection and isolation of faults in PVTOLs. An approach to fault isolation for a PVTOL based on nonlinear decoupling is reported by Rodríguez Alfaro (2014).

In this paper an approach to fault detection and isolation in a wide class of nonlinear systems, i.e., for systems which allow a Hamiltonian representation, is considered. For this class of systems it is always possible to design an observer-based residual. Using this particular property, decoupled subsystems are defined and represented now as Hamiltonian systems. In consequence, it is always possible to obtain a solution to the fault detection problem. A Hamiltonian representation is derived from an Euler-Lagrange representation of the PVTOL and the proposed approach applied. As a result, the faults considered can be detected and a different set of faults (not every fault) can be isolated. In contrast to a geometric approach (DePersis and Isidori, 2001), the approach considered keeps the observer design realizable. Simulations are used to illustrate these results. 
The paper is organized as follows. In the next section the Hamiltonian approach to fault diagnosis is presented, in Section 3 the PVTOL system is introduced, in Section 4 an application of the proposed approach is considered and some results are discussed. Conclusions are presented in Section 5.

\section{Hamiltonian approach to fault diagnosis}

2.1. Fault detection and isolation. There are a lot of publications on fault detection and isolation of linear systems (see, e.g., Chen and Patton, 1999; Blanke et al., 2006; Ding, 2013). For nonlinear systems there are also some solutions for different classes of systems (Alcorta-Garcia and Frank, 1997; Zhang and Jiang, 2008). DePersis and Isidori (2001) propose a geometric approach. Most of the approaches are for specific nonlinear structures; however, when a general case is considered, they have some limited existence conditions.

From an ideal point of view, residuals are signals which only depend on faults and they are ideally zero when no fault is present (Frank, 1990). Once residuals are available, a residual evaluation function is required to extract the fault information from the residuals. Generally, a threshold is needed in the residual evaluation in order to avoid false alarms. Note that residuals should only depend on faults, but in reality this will never happen. It should be seen more like a design specification and not as a fact (Ding, 2013). A general schema for fault detection used here is represented in Fig. 1

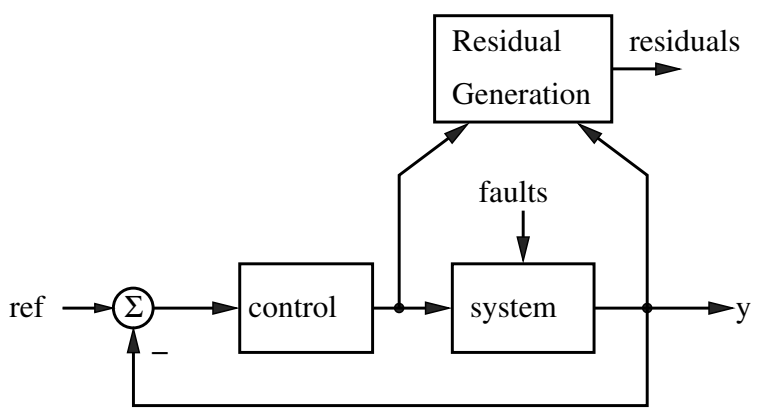

Fig. 1. General schema for residual generation.

2.2. Hamiltonian approach to fault diagnosis. In this work a novel fault detection and isolation algorithm, the so-called Hamiltonian approach to fault diagnosis, is considered. It has been recently proposed by Rodríguez Alfaro (2014) and is inspired by the well-known solution to the fault detection and isolation problem, i.e., it follows a classical way to fault detection and isolation (Frank, 1990): first a decoupling of different faults (or groups of faults) is realized; after that, a residual is obtained using an observer for the decoupled subsystem (subsystems with specific fault sensitivity). For residual generation, an observer based design can be used. The evaluation of each residual brings information also on fault isolation. In contrast to that classical approach, the proposed Hamiltonian schema takes advantage of the structure of the Hamiltonian representation and allows the design of the residual generation based on the structure also for nonlinear systems. The different steps of fault diagnosis in Hamiltonian systems are the following:

1. representation of the system in the generalized Hamiltonian form,

2. calculation of the subsystems sensible to a specific fault,

3. design of an observer-based residual generator for the decoupled subsystem,

4. evaluation function to decide when and where a fault is occurring.

Each of the steps will be described in detail in the following subsections.

\subsection{Generalized Hamiltonian representation.} Consider a nonlinear systems described by

$$
\begin{aligned}
\dot{\bar{x}} & =f(\bar{x}, u), \\
y & =h(\bar{x}),
\end{aligned}
$$

where $\bar{x} \in \mathbb{R}^{n}$ is the state vector, $u \in \mathbb{R}^{p}$ is the input vector and $y \in \mathbb{R}^{m}$ represents the output vector, $f(\cdot, \cdot)$ is a vector field, continuous with respect to its arguments.

A smooth nonlinear system (1) can be represented in a generalized Hamiltonian form (Sira Ramírez and Cruz Hernández, 2001; van der Schaft, 2000), which is given in the following equation:

$$
\dot{x}=[J(x)+S(x)] \frac{\partial H(x)}{\partial x}+F(x)+G(u),
$$

with $x \in \mathbb{R}^{n}$ being the state vector, $u$ the input vector, $H(x)$ a smooth energy function, globally positive definite in $\mathbb{R}^{n}$. A possible form for $H(x)$ is given by

$$
H(x)=\frac{1}{2} x^{T} M x,
$$

where $M$ is a constant matrix which is positive definite. Taking the derivative of $H(x)$ with respect to $x$, a gradient results in a vector $\partial H / \partial x=M x$. The matrix $J(x)$ represents a conservative part of the system and matrix $S(x)$ represents a non conservative part of the system. Both matrices should satisfy, for every $x \in \mathbb{R}^{n}$, the following conditions:

$$
\begin{aligned}
& J(x)=-J^{T}(x), \\
& S(x)=S^{T}(x),
\end{aligned}
$$


and $F(x)$ represents a locally unstable vector field.

Many physical systems admit a representation in a generalized Hamiltonian form, like electromechanical systems, electrical systems, mechanical systems, and others.

Note that additive faults in the generalized Hamiltonian representation appear as additional inputs (unknown inputs)

$$
\begin{aligned}
\dot{x}= & J(x) \frac{\partial H}{\partial x}+S(x) \frac{\partial H}{\partial x} \\
& +F(x)+G(u)+N(\Delta f), \\
y= & C \frac{\partial H}{\partial x}+Q(\Delta f),
\end{aligned}
$$

where $N(\Delta f)$ and $Q(\Delta f)$ represent additive faults of the system.

2.4. Fault decoupling. The main idea is to define a set of transformations in such a way that each transformed system (corresponding to each state transformation) depends on a specific set of faults (or of a single fault). There are some works on the analysis and synthesis of these transformations (e.g., Seliger and Frank, 1991; Alcorta Garcia, 1999; DePersis and Isidori, 2001). The basic idea used by Seliger and Frank (1991) is to find a fault decoupled system.

Consider a system with faults in Eqn. (5) and a nonlinear transformation

$$
\zeta=T(x)
$$

It is required that

$$
\begin{aligned}
\dot{\zeta}= & \frac{\partial T(x)}{\partial x} \dot{x} \\
= & \frac{\partial T(x)}{\partial x}\left[J(x) \frac{\partial H(x)}{\partial x}+S(x) \frac{\partial H(x)}{\partial x}+F(x)\right. \\
& +G(u)+N(\Delta f)]
\end{aligned}
$$

and the transformation $T(x)$ should be selected in such a way that the resulting transformed system has the desired fault sensibility, i.e., suppose $N(\Delta f)=$ $\left[n_{1}(\Delta f) n_{2}(\Delta f) \cdots n_{l}(\Delta f)\right]$, where $\bar{N}(\Delta f)$ represent the columns associated with the faults that are required not to affect a specific subsystem and $\overline{\bar{N}}(\Delta f)$ are the columns related to the faults that are required to affect the subsystem, with

$$
\frac{\partial T(x)}{\partial x} \bar{N}(\Delta f)=0, \quad \frac{\partial T(x)}{\partial x} \overline{\bar{N}}(\Delta f) \neq 0 .
$$

In the work of Seliger and Frank (1991), details about the existence of this transformation can be found.
2.5. Observer for generalized Hamiltonian systems. An observer for the class of nonlinear systems called generalized Hamiltonian has been proposed by Sira Ramírez and Cruz Hernández (2001). Some modifications have been added in order to consider an extension for the case where the vector function $F$ depends on the state and not on the output like in the work of Sira Ramírez and Cruz Hernández (2001). Instead, the proposed observer is more like a Thau observer (Thau, 1973).

Consider a generalized Hamiltonian system as represented by (2) with its corresponding output $y$ :

$$
\begin{aligned}
\dot{x}= & {[J(y)+S(y)] \frac{\partial H(x)}{\partial x} } \\
& +F(x)+G(u), \quad x \in \mathbb{R}^{n}, \\
y= & C \frac{\partial H(x)}{\partial x}, \quad y \in \mathbb{R}^{m},
\end{aligned}
$$

where $y$ is the output vector, $C$ is a constant output matrix of an appropriate dimension.

An observer for the system (8) is defined by

$$
\begin{aligned}
\dot{\xi}= & {[J(y)+S(y)] \frac{\partial H(\xi)}{\partial \xi} } \\
& +F(\xi)+G(u)+K(y-\eta), \\
\eta= & C \frac{\partial H(\xi)}{\partial \xi},
\end{aligned}
$$

with $K$ being the observer gain, $\xi$ the estimated state, $\eta$ the estimated output, and $\partial H(\xi) / \partial \xi=M \xi$ the gradient vector, with $M$ being a positive definite matrix.

The corresponding state estimation error, defined by $e(t)=x(t)-\xi(t)$, as well as the output estimation error $e_{y}(t)=y(t)-\eta(t)$, are given by

$$
\begin{aligned}
\dot{e}= & J(y) \frac{\partial H(e)}{\partial e}+[S-K C] \frac{\partial H(e)}{\partial e} \\
& +\tilde{F}(x, \xi), \quad e \in \mathbb{R}^{n}, \\
e_{y}= & C \frac{\partial H(e)}{\partial e}, \quad e_{y} \in \mathbb{R}^{m},
\end{aligned}
$$

where $\partial H(e) / \partial e$ is a gradient vector of the modified energy function

$$
\frac{\partial H(e)}{\partial e}=\frac{\partial H(x)}{\partial x}-\frac{\partial H(\xi)}{\partial \xi}=M(x-\xi)=M e .
$$

The design conditions are described in the following theorem.

Theorem 1. The state $x$ of the nonlinear system (8) can be globally, exponentially, asymptotically estimated by the system (9) if the pair $(C, S)$ is observable or at least detectable and the matrix

$$
M^{T}\left[S-\frac{1}{2}\left(K C+C^{T} K^{T}\right)\right] M+\Pi
$$


is negative definite, with

$$
\Pi=\frac{1}{2}\left[M \frac{\partial F}{\partial x}(\zeta)+\left(\frac{\partial F}{\partial x}(\zeta)\right)^{T} M^{T}\right]
$$

and $\zeta$ being a vector such that $\zeta \in(x, \xi)$.

Proof. From the generalized Hamiltonian representation

$$
\begin{aligned}
& \dot{x}=J \frac{\partial H(x)}{\partial x}+S \frac{\partial H(x)}{\partial x}+F(x)+G(u), \\
& y=C \frac{\partial H(x)}{\partial x}
\end{aligned}
$$

and considering the observer described by

$$
\begin{aligned}
\dot{\hat{x}}= & J \frac{\partial H(\hat{x})}{\partial \hat{x}}+S \frac{\partial H(\hat{x})}{\partial \hat{x}} \\
& +F(\hat{x})+G(u)+K(y-\eta), \\
\eta= & C \frac{\partial H(\hat{x})}{\partial \hat{x}}
\end{aligned}
$$

defining the error as $e=x-\hat{x}$ and $\dot{e}=\dot{x}-\dot{\hat{x}}$, and replacing $\dot{x}$ and $\dot{\hat{x}}$ into $\dot{e}$, results in

$$
\dot{e}=J \frac{\partial H}{\partial e}+(S-K C) \frac{\partial H}{\partial e}+F(x)-F(\hat{x}),
$$

where

$$
\frac{\partial H}{\partial e}=\frac{\partial H}{\partial x}-\frac{\partial H}{\partial \hat{x}}=M(x-\hat{x})=M e .
$$

Taking as a modified Hamiltonian energy function the positive definite function

$$
H(e)=\frac{1}{2} e^{T} e,
$$

with the time derivative

$$
\dot{H}(e)=\frac{\partial H(e)}{\partial e} \dot{e},
$$

and replacing $\dot{e}$ in 16 , we obtain

$$
\begin{aligned}
\dot{H}(e)= & \frac{\partial H(e)}{\partial e}(J+S-K C) \frac{\partial H(e)}{\partial e} \\
& +\frac{\partial H(e)}{\partial e}(F(x)-F(\dot{\hat{x}})) .
\end{aligned}
$$

Since $J$ is a skew symmetric matrix, 17) is reduced to

$$
\begin{aligned}
\dot{H}(e)= & \frac{\partial H(e)}{\partial e}\left[S-\frac{1}{2}\left(K C+C^{T} K^{T}\right)\right] \frac{\partial H(e)}{\partial e} \\
& +\frac{\partial H(e)}{\partial e}(F(x)-F(\dot{\hat{x}}))
\end{aligned}
$$

where the skew symmetric part of the matrix $K C$ is eliminated. Now, applying the mean-value theorem (see, for example, Apostol, 1967) to the last term of $(18)$, we have

$$
F(x)-F(\hat{x})=\frac{\partial F(\rho)}{\partial x} e .
$$

Finally, it is obtained that

$$
\begin{aligned}
& \dot{H}(e) \\
& =e^{T}\left[M^{T}\left(S-\frac{1}{2}\left(K C+C^{T} K^{T}\right)\right) M+\Pi\right] e
\end{aligned}
$$

where

$$
\Pi=\frac{1}{2}\left(M^{T} \frac{\partial F(\rho)}{\partial x}+{\frac{\partial F(\rho)^{T}}{\partial x}}^{T} M\right) .
$$

For (20) with the condition

$$
\begin{array}{r}
\exists K \mid \lambda\left(M^{T}\left(S-\frac{1}{2}\left(K C+C^{T} K^{T}\right)\right) M+\Pi\right) \in \mathbb{C}_{-} \\
\Rightarrow \quad \dot{H}(e)<0,
\end{array}
$$

it is guaranteed that the estimation error e tends to zero as the time tends to infinity.

\section{Planar vertical take-off and landing model}

The PVTOL model of an aircraft consists of two rotors attached to a rigid bar with the vehicle mass center, at the same distance from each other (see Fig. 2).

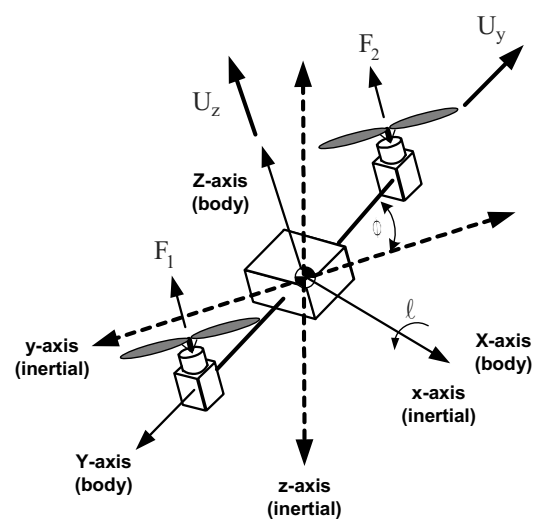

Fig. 2. Two rotor PVTOL.

This paper has not considered the uncertainty in the model. However, the main source of uncertainty is with the measurement of the variables: position and angles. The mass of the PVTOL can be also a source of uncertainties, because of the way to pass energy to the motors.

A mathematical model can be developed using the flat-Earth model equations to represent the kinematics, 
position, forces and moments (Stevens and Lewis, 2003). Details for obtaining the mathematical model of the PVTOL can be found, e.g., in the works of Castillo et al. (2004), as well as Etkin and Reid (1996) and Murray et al. (1994).

The system model of a PVTOL can be written in the standard form

$$
D(q) \ddot{q}+G(q)=\tau .
$$

This is a second-order differential equation for the motion of an under-actuated system, where $\tau$ is the forces and moments input vector, $G(q)$ includes gravity terms, the matrix $D(q)$ summarizes the inertia properties.

Then, defining $q \triangleq\left[\begin{array}{ccc}Y & Z & \phi\end{array}\right]$ as the generalized state vector, $Y$ is the displacement in the $Y$-axis, $Z$ the displacement in the $Z$-axis and $\phi$ the angle related to the $X$-axis, and the input vector $\tau=\left[\begin{array}{lll}U_{z} & U_{y} & l\end{array}\right]$ for the PVTOL, where $l$ is the rolling moment, the lateral force $U_{y}$ is related to the rolling moment $l$ by $U_{y}=\epsilon_{0} l$, the term $\epsilon_{0}$ represents the transport acceleration and characterizes the coupling between the angular momentum and the angular acceleration of the vehicle and $U_{z}$ is the thrust force in the $Z$-axis:

$$
\begin{aligned}
& D(q)=\left[\begin{array}{ccc}
-m \sin (\phi) & m \cos (\phi) & 0 \\
m \cos (\phi) & m \sin (\phi) & 0 \\
0 & 0 & J_{x}
\end{array}\right], \\
& G(q)=\left[\begin{array}{c}
m g \cos (\phi) \\
m g \sin (\phi) \\
0
\end{array}\right],
\end{aligned}
$$

where $m$ represent the mass, $g$ is the gravity force, $J_{x}$ is the inertia. Note that the PVTOL model (22) is a representation based on an Euler-Lagrange formalism (Murray et al., 1994).

\section{Hamiltonian approach to fault diagnosis in PVTOLs}

In order to take advantage of the observer presented in Section 2.5, a generalized Hamiltonian representation is required.

4.1. Hamiltonian representation of PVTOLs. Using the state transformation given by van der Schaft (2000, Chapter 4), the PVTOL system (22) can be represented by Eqns. (8). Let us consider a generalized moment (van der Schaft, 2000) $p(t)$ defined by

$$
p \triangleq D(q) \dot{q}
$$

as well as the Hamiltonian function $H$ given by

$$
H(q, p)=\frac{1}{2} p^{T} D^{-1}(q) p+P_{E}(q)
$$

with $P_{E}(q)$ being the potential energy term of the PVTOL.

The system 22 can be alternatively represented by

$$
\begin{aligned}
& \dot{q}=\frac{\partial H(q, p)}{\partial p}=D^{-1}(q) p, \\
& \dot{p}=-\frac{\partial H(q, p)}{\partial q}+\tau, \\
& \dot{p}=-\frac{\partial}{\partial q}\left(\frac{1}{2} p^{T} D^{-1}(q) p\right)-\frac{\partial}{\partial q} P_{E}(q)+\tau,
\end{aligned}
$$

where the well-known relation

$$
\frac{\partial P_{E}(q)}{\partial q}=G(q)
$$

is used (see, for example, Ortega et al., 1998). The PVTOL equations are as follows:

$$
\begin{aligned}
\dot{x}_{1}= & -\frac{x_{4}}{m} \sin \left(x_{3}\right)+\frac{x_{5}}{m} \cos \left(x_{3}\right), \\
\dot{x}_{2}= & \frac{x_{4}}{m} \cos \left(x_{3}\right)+\frac{x_{5}}{m} \sin \left(x_{3}\right), \\
\dot{x}_{3}= & \frac{x_{6}}{J_{x}}, \\
\dot{x}_{4}= & -m g \cos \left(x_{3}\right)+U_{z}, \\
\dot{x}_{5}= & -m g \sin \left(x_{3}\right)+U_{y}, \\
\dot{x}_{6}= & \frac{x_{4}^{2} \cos \left(x_{3}\right)}{2 m}+\frac{x_{4} x_{5} \sin \left(x_{3}\right)}{m} \\
& -\frac{x_{5}^{2} \cos \left(x_{3}\right)}{2 m}+l,
\end{aligned}
$$

where $x^{T}=\left[\begin{array}{llllll}x_{1} & x_{2} & x_{3} & x_{4} & x_{5} & x_{6}\end{array}\right]$ and

$$
x^{T}=\left[\begin{array}{llllll}
Y & Z & \phi & p_{1} & p_{2} & p_{3}
\end{array}\right]=\left[\begin{array}{ll}
q^{T} & p^{T}
\end{array}\right] .
$$

Now, defining a Hamiltonian $\mathcal{H} \triangleq \frac{1}{2} x^{T} x$ in order to arrive at the form (2), the following equations are obtained:

$$
\begin{aligned}
& \frac{\partial \mathcal{H}}{\partial x}=x, \quad M=I \in \mathbb{R}^{6 \times 6}, \\
& J(x)=[0] \in \mathbb{R}^{6 \times 6}, \quad S(x)=[0] \in \mathbb{R}^{6 \times 6}, \\
& F(x)
\end{aligned}
$$

$$
\begin{gathered}
-\frac{x_{4} \sin \left(x_{3}\right)}{m}+\frac{x_{5} \cos \left(x_{3}\right)}{m} \\
\frac{x_{4} \cos \left(x_{3}\right)}{m}+\frac{x_{5} \sin \left(x_{3}\right)}{m} \\
\frac{x_{6}}{J_{x}} \\
-m g \cos \left(x_{3}\right) \\
\frac{-m g}{\sin \left(x_{3}\right)} \\
G^{T}=\left[\begin{array}{cccccc}
0 \\
\frac{x_{4}^{2} \cos \left(x_{3}\right)}{2 m}+\frac{x_{4}}{x_{5}} \sin \left(x_{3}\right) \\
0 & 0 & 0 & 1 & 0 & 0 \\
0 & 0 & 0 & 0 & 1 & 0 \\
0 & 0 & 0 & 0 & 0 & 1
\end{array}\right] .
\end{gathered}
$$


The Hamiltonian representation results in the following equation:

$$
\begin{aligned}
& \dot{x}=J(x) \frac{\partial \mathcal{H}}{\partial x}+S(x) \frac{\partial \mathcal{H}}{\partial x}+F(x)+G \underbrace{\left[\begin{array}{c}
U_{z} \\
U_{y} \\
l
\end{array}\right]}_{u}, \\
& y=C \frac{\partial \mathcal{H}}{\partial x},
\end{aligned}
$$

with

$$
C=\left[\begin{array}{llllll}
1 & 0 & 0 & 0 & 0 & 0 \\
0 & 1 & 0 & 0 & 0 & 0 \\
0 & 0 & 1 & 0 & 0 & 0
\end{array}\right]
$$

4.2. Fault modeling. In this work five faults are considered: one for each sensor and one for each actuator. Faults could be multiplicative or additive, depending on the way they appear in the equations. Additive faults are like an external input to the system or to the output equations. Multiplicative faults are in product with states, inputs or both. Depending on the form in which the faults are manifested, they could be incipient, abrupt or intermittent (Chen and Patton, 1999; Isermann, 2006; Ding, 2008).

For each sensor and actuator, additive and multiplicative faults are considered. However, due to the space conditions, only abrupt faults are tested.

4.2.1. Additive fault representation. Additive faults are implemented as an additive input in each actuator channel $\left(f_{a i}\right)$ as well as in each output channel $\left(f_{s i}\right)$ :

$$
\begin{aligned}
& \dot{x}=(J(x)+S(x)) \frac{\partial \mathcal{H}}{\partial x}+F(x)+G u+G_{f}\left[\begin{array}{l}
f_{a 1} \\
f_{a 2} \\
f_{a 3}
\end{array}\right], \\
& y=C \frac{\partial \mathcal{H}}{\partial x}+\left[\begin{array}{c}
f_{s 1} \\
f_{s 2} \\
f_{s 3}
\end{array}\right],
\end{aligned}
$$

where

$$
G_{f}^{T}=\left[\begin{array}{cccccc}
0 & 0 & 0 & 1 & 0 & 0 \\
0 & 0 & 0 & 0 & 1 & 0 \\
0 & 0 & 0 & 0 & 0 & 1
\end{array}\right]
$$

The faults have been modeled as step functions. The time at which the fault occurs is the time for the step. In general, additive faults are a bias for the sensors and/or actuators. Different magnitudes will be tested in the fault detection scheme.

4.2.2. Multiplicative fault representation. The case of a multiplicative fault representation corresponds to a parameter change in the system. Actuator faults are actually in product with the input; multiplicative sensor faults are in product with the state. Multiplicative faults are represented as $\Delta f_{a i}$ for actuators and $\Delta f_{s i}$ for sensors. The system with multiplicative faults is as follows:

$$
\begin{aligned}
\dot{x}= & (J(x)+S(x)) \frac{\partial \mathcal{H}}{\partial x}+F(x)+G u \\
& +G_{\Delta f}(u)\left[\begin{array}{c}
\Delta f_{a 1} \\
\Delta f_{a 2} \\
\Delta f_{a 3}
\end{array}\right], \\
y= & C \frac{\partial \mathcal{H}}{\partial x}+C_{\Delta f}(x)\left[\begin{array}{l}
f_{s 1} \\
f_{s 2} \\
f_{s 3}
\end{array}\right],
\end{aligned}
$$

where

$$
\begin{aligned}
G_{\Delta f}^{T}(u) & =\left[\begin{array}{cccccc}
0 & 0 & 0 & U_{z} & 0 & 0 \\
0 & 0 & 0 & 0 & U_{y} & 0 \\
0 & 0 & 0 & 0 & 0 & l
\end{array}\right], \\
C_{\Delta f}^{T}(x) & =\left[\begin{array}{cccccc}
x_{1} & 0 & 0 & 0 & 0 & 0 \\
0 & x_{2} & 0 & 0 & 0 & 0 \\
0 & 0 & x_{3} & 0 & 0 & 0
\end{array}\right] .
\end{aligned}
$$

4.3. Fault decoupling. A kind of generalized observer scheme (Frank, 1990) will be used here. The general strategy is to make a decoupling in order to obtain a subsystem robust to each of the possible fault origins, i.e., a sensor and an actuator, one by one.

4.3.1. Sensor faults. For sensor faults, only one sensor is considered as the output of the whole system. For a simpler model for the subsystem 1, state $x_{3}$ is substituted using $y_{3}$, i.e., the sensor fault 3 will also affect the subsystem 1 . It is also necessary to test which of the actuator faults also affects the subsystem. An observer-based residual is designed for the corresponding observable part. The separation of the observable part of the system could be a laborious task. However, in any event, for the sensor faults, a full-order observer could be designed. The output estimation error will be used as a residual, so three residuals are obtained. Therefore, each residual can be associated to each fault.

Subsystem 1. For the first sensor, under the assumption that $x_{3}$ is reliable (or available from the output $y_{3}$ ), a subsystem could be formed by Eqns. (28), (31) and (32). Note that for this subsystem the design is simple:

$$
\begin{aligned}
\dot{x}_{1} & =-\frac{x_{4}}{m} \sin \left(x_{3}\right)+\frac{x_{5}}{m} \cos \left(x_{3}\right), \\
\dot{x}_{4} & =-m g \cos \left(x_{3}\right)+U_{z}, \\
\dot{x}_{5} & =-m g \sin \left(x_{3}\right)+U_{y}, \\
y_{s 1} & =x_{1} .
\end{aligned}
$$

Subsystem 2. For the second sensor, let us consider Eqns. (29), (31) and (32). Again, the measured third state 
$x_{3}$ is assumed as reliable:

$$
\begin{aligned}
\dot{x}_{2} & =\frac{x_{4}}{m} \cos \left(x_{3}\right)+\frac{x_{5}}{m} \sin \left(x_{3}\right), \\
\dot{x}_{4} & =-m g \cos \left(x_{3}\right)+U_{z}, \\
\dot{x}_{5} & =-m g \sin \left(x_{3}\right)+U_{y}, \\
y_{s 2} & =x_{2} .
\end{aligned}
$$

Subsystem 3. For the third sensor, which corresponds to $x_{3}$, the subsystem is formed by (30), 31, 32) and (33):

$$
\begin{aligned}
\dot{x}_{3} & =\frac{x_{6}}{J_{x}} \\
\dot{x}_{6} & =\frac{x_{4}^{2}}{2 m} \cos \left(x_{3}\right)+\frac{x_{4} x_{5}}{m} \sin \left(x_{3}\right)-\frac{x_{5}^{2}}{2 m} \cos \left(x_{3}\right)+l, \\
\dot{x}_{4} & =-m g \cos \left(x_{3}\right)+U_{z} \\
\dot{x}_{5} & =-m g \sin \left(x_{3}\right)+U_{y} \\
y_{s 3} & =x_{3} .
\end{aligned}
$$

If the measurement of $x_{3}$ is not reliable, $f_{s 3}$ will also affect subsystems associated with the sensor faults 1 and 2.

4.3.2. Actuator faults. In order to isolate the actuator faults, a decoupling strategy is required. Each case will be discussed.

Subsystem 4. For the first actuator fault $\left(f_{a 1}\right.$ and/or $\left.\Delta f_{a 1}\right)$, it is possible to follow this reasoning: Because the fault for which the residual should be sensible is in the same channel as $U_{z}$, take (31) together with 28), because $x_{1}$ is measured. Using the fact that $x_{3}$ is also available from the output, we can make an observer-based residual for this subsystem using the output estimation error $\left(r_{1 a}=y_{1}-\hat{y}_{1}\right)$ as a residual. Then $x_{5}$ should be obtained from 29]. The subsystem 4 results in

$$
\begin{aligned}
\dot{x}_{1} & =-\frac{x_{4}}{m \sin \left(x_{3}\right)}+\frac{\dot{x}_{2} \cos (x 3)}{\sin \left(x_{3}\right)}, \\
\dot{x}_{4} & =-m g \cos \left(x_{3}\right)+U_{z}, \\
y_{a 1} & =x_{1} .
\end{aligned}
$$

Subsystem 5. For the second actuator fault $f_{a 2}$ and/or $\Delta f_{a 2}$ we use the actuators (28) and (32), assuming that $x_{3}$ is available in the measurable output $y_{3}$. Since $x_{4}$ is not measurable, its value results from Eqn. 29). Then, the value of $x_{4}$ is substituted in (28) and, considering (32), the subsystem 5 is obtained:

$$
\begin{aligned}
\dot{x}_{1} & =\frac{x_{5}}{m \cos \left(x_{3}\right)}-\frac{\dot{x}_{2} \sin (x 3)}{\cos \left(x_{3}\right)}, \\
\dot{x}_{5} & =-m g \sin \left(x_{3}\right)+U_{y}, \\
y_{a 2} & =x_{1}
\end{aligned}
$$

Subsystem 6. For the third actuator fault $f_{a 3}$ and/or $\Delta f_{a 3}$ Eqns. (30) and (33) are used to get the subsystem
6. Since Eqn. (33) depends on $x_{4}$ and $x_{5}$, which are not measurable, its values are obtained from the other equations. The value of $x_{5}$ is obtained solving Eqn. (28), the value of $x_{4}$ is substituted in (29) and the resulting equation is solved for $x_{5}$. Now, Eqn. (28) is solved for $x_{5}$, the result is substituted in (29) and the resulting equation is solved for $x_{4}$ :

$$
\begin{aligned}
\dot{x}_{3} & =\frac{x_{6}}{J_{x}} \\
\dot{x}_{6} & =\frac{x_{4}^{2}}{2 m} \cos \left(x_{3}\right)+\frac{x_{4} x_{5}}{m} \sin \left(x_{3}\right)-\frac{x_{5}^{2}}{2 m} \cos \left(x_{3}\right)+l, \\
y_{a 3} & =x_{3},
\end{aligned}
$$

where

$$
\begin{aligned}
& x_{4}=m \cos (x 3)\left(\dot{x}_{2}-\frac{\dot{x}_{1} \sin (x 3)}{\cos (x 3)}\right), \\
& x_{5}=m \sin (x 3)\left(\dot{x}_{2}-\frac{\dot{x}_{1} \cos (x 3)}{\sin (x 3)}\right) .
\end{aligned}
$$

Note that the time derivatives of $x_{2}$ and $x_{1}$, i.e., of $y_{2}$ and $y_{1}$, will be required. A numerical approximation is used in this work. Some recent results (Vasiljevic and Khalil, 2008) can be also applied to obtain estimates of the time derivatives.

4.4. Observer-based residual design. The observer-based residuals are designed as presented in Section 2.5. For the subsystems sensible to sensor faults, the design is simple. As an example, residual generator design with details for the sensor 1 is presented.

Consider the first subsystem:

$$
\begin{aligned}
& \dot{x}_{1}=-\frac{x_{4}}{m} \sin \left(x_{3}\right)+\frac{x_{5}}{m} \cos \left(x_{3}\right), \\
& \dot{x}_{4}=-m g \cos \left(x_{3}\right)+U_{z}, \\
& \dot{x}_{5}=-m g \sin \left(x_{3}\right)+U_{y}, \\
& y_{1}=x_{1} .
\end{aligned}
$$

Consider the observer-based residual generator:

$$
\begin{aligned}
\dot{\hat{x}}_{1} & =-\frac{\hat{x}_{4}}{m} \sin \left(x_{3}\right)+\frac{\hat{x}_{5}}{m} \cos \left(x_{3}\right)+L_{1}\left(y_{1}-\hat{x}_{1}\right), \\
\dot{\hat{x}}_{4} & =-m g \cos \left(x_{3}\right)+U_{z}, \\
\dot{\hat{x}}_{5} & =-m g \cos \left(x_{3}\right)+U_{y}, \\
r_{1} & =y_{1}-\hat{x}_{1} .
\end{aligned}
$$

Define the estimation errors as

$$
\begin{gathered}
e_{1} \triangleq x_{1}-\hat{x}_{1}, \\
e_{4} \triangleq x_{4}-\hat{x}_{4}, \\
e_{5} \triangleq x_{5}-\hat{x}_{5} .
\end{gathered}
$$


The estimation error dynamics are

$$
\begin{aligned}
\dot{e}_{1} & =\frac{e_{5} \cos \left(x_{3}\right)-e_{4} m \sin \left(x_{3}\right)}{m}-L_{1} e_{1}, \\
\dot{e}_{4} & =-e_{4}, \\
\dot{e}_{5} & =-e_{5}, \\
r_{1} & =e_{1} .
\end{aligned}
$$

Note that $r_{1}$ goes to zero when time increases if no faults ( $f_{1}$ and/or $f_{3}$ ) are present in the PVTOL system.

Residual design for faults in the second sensor (second subsystem) is similar to the one above, but we change the differential equation of $\dot{x}_{1}$ by the corresponding one of $\dot{x}_{2}$. The details of the design will not be presented here.

The subsystem sensible to actuator faults in the input channel of $U_{z}$ results in a subsystem given by

$$
\begin{aligned}
\dot{x}_{1} & =\frac{-x_{4}}{m \sin \left(x_{3}\right)}+\frac{\dot{y}_{2} \cos \left(x_{3}\right)}{\sin \left(x_{3}\right)}, \\
\dot{x}_{4} & =-m g \cos \left(x_{3}\right)+U_{z}, \\
y_{4} & =x_{4},
\end{aligned}
$$

which can be written in the form (2) with

$$
\begin{aligned}
J(x) & =[0] \in \mathbb{R}^{2 \times 2}, \\
S(x) & =[0] \in \mathbb{R}^{2 \times 2}, \\
F(x) & =\left[\begin{array}{c}
\frac{m \dot{y}_{2} \cos \left(x_{3}\right)-x_{4}}{m \sin \left(x_{3}\right)} \\
-m g \cos \left(x_{3}\right)
\end{array}\right], \\
G & =\left[\begin{array}{l}
0 \\
1
\end{array}\right], \\
C & =\left[\begin{array}{ll}
1 & 0
\end{array}\right] .
\end{aligned}
$$

In the above subsystem, the pair $(C, S)$ is detectable. Therefore, an observer-based residual can be designed. The residual is designed using a copy of the subsystem (model) and a correction factor defined by $L\left(y_{4}-\hat{y}_{4}\right)$, with the observer gain $L^{T}=\left[\begin{array}{ll}L_{4} & 0\end{array}\right]$.

For the second actuator fault $\left(f_{a 2}, \Delta f_{a 2}\right)$, for the third actuator fault $\left(f_{a 3}, \Delta f_{a 3}\right)$ and for the fault in the third sensor $\left(f_{s 3}, \Delta f_{s 3}\right)$, the decoupled required subsystem is similar to the procedure used for the actuator fault $f_{a 1}$. Table 1 presents a summary of the different fault sensitivity, where $\checkmark$ means that the subsystem presents sensibility to the corresponding fault, i.e., the fault affects the corresponding subsystem.
Table 1. Subsystems $\left(\sum_{\text {mb }}\right)$ sensitive to different faults.

\begin{tabular}{|c|c|c|c|c|c|c|}
\hline Faults & $\Sigma_{\text {sub }}$ & 2 & 3 & 4 & 5 & 6 \\
\hline \hline$f_{s 1}$ & $\checkmark$ & & & $\checkmark$ & $\checkmark$ & $\checkmark$ \\
\hline$f_{s 2}$ & & $\checkmark$ & & $\checkmark$ & & $\checkmark$ \\
\hline$f_{s 3}$ & $\checkmark$ & $\checkmark$ & $\checkmark$ & $\checkmark$ & $\checkmark$ & $\checkmark$ \\
\hline$f_{a 1}$ & & $\checkmark$ & $\checkmark$ & $\checkmark$ & & \\
\hline$f_{a 2}$ & $\checkmark$ & & $\checkmark$ & & $\checkmark$ & \\
\hline$f_{a 3}$ & & & $\checkmark$ & & & $\checkmark$ \\
\hline
\end{tabular}

From Table 1 it results clearly that the subsystems 1 and 5 have the same fault sensitivity $\left\{f_{s 1}, f_{s 3}, f_{a 2}\right\}$, so one of them is unnecessary.

Note that five residuals are obtained for the PVTOL under the assumption that only a single fault occurred at one time (given by the observer-based approach applied to the subsystems $\{1,2,3,4,6\}$ or $\{1,2,3,4,5\})$.

Formally, for fault isolation, i.e., the isolation of the six single faults considered, only three residuals are required. Note that the different sets of three residuals associated with the corresponding subsystems that can be used are, e.g. $\{1,2,3\},\{1,2,6\},\{1,3,6\},\{1,3,4\}$, $\{2,3,6\}$. Each of them could be used for the isolation of the six faults. However, the set of residuals associated with $\{1,4,6\}$ will isolate only five faults. Further, under the last set of residuals, $f_{s 1}$ and $f_{s 3}$ appear coupled. The other set of residuals inadequate for the isolation of all the faults considered is $\{1,2,4\}$. For this set of residuals only four groups of faults (three of them are single faults), $f_{s 1}, f_{s 3}$, $f_{a 2}$ and the group $\left\{f_{s 2}, f_{a 1}\right\}$ can be isolated. The fault $f_{a 3}$ has no effect on this set of residuals.

Remark 1. A factor to be considered for the selection of the set of residuals (i.e., a corresponding subsystem associated with a specific fault sensitivity) is also to observe that some of the residuals obtained in Section 4.3 have the requirements of time derivatives. In the case of the subsystems obtained, three of them require time derivatives. Actually, the three residuals obtained were inspired by acquisition of a subsystem robust to a specific actuator fault. Obtaining time derivatives of the system output could be a hard task if noise is present.

Observer-based residual generation for the first three subsystems seems to be the more robust option. The effect of noise is reduced because of filter characteristics of the observer.

4.5. Simulation results. The following parameters are used in the simulation results: mass $m=0.8 \mathrm{~kg}$, gravity constant $g=9.81 \mathrm{~m} / \mathrm{sec}^{2}$ and inertia $J_{x}=0.015 \mathrm{~kg} \mathrm{~m}^{2}$. For all faulty cases a fault magnitude equal to 1 , occurring at time $t=75 \mathrm{sec}$, was considered.

Figure 3 shows the output signals of the PVTOL without failures. Figure 4 shows the state signals of the 


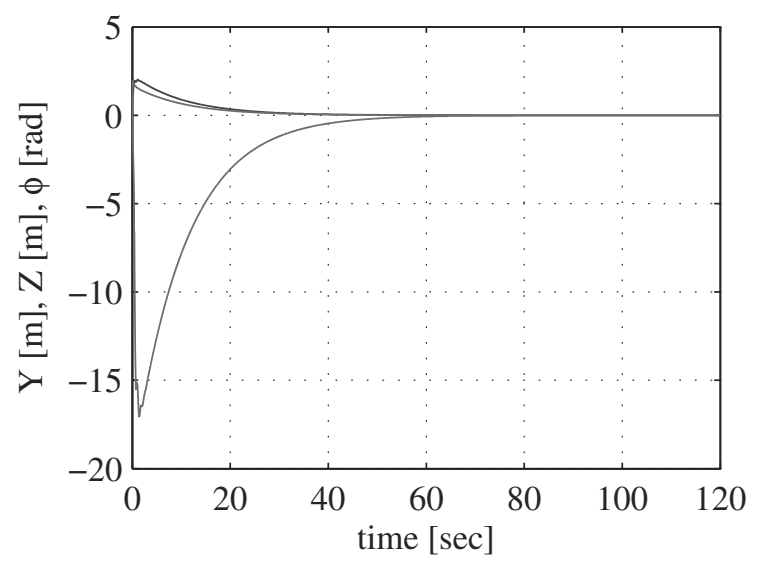

Fig. 3. PVTOL output signals in nominal conditions.

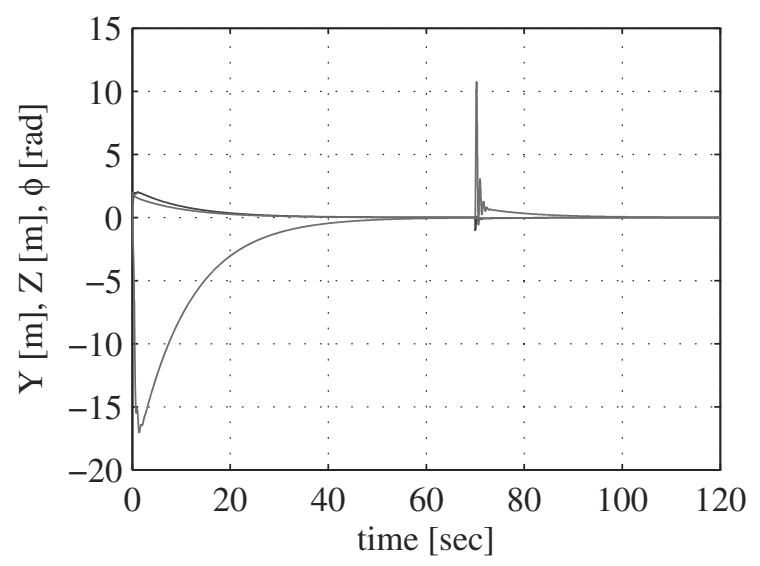

Fig. 4. PVTOL output signals when the fault $f_{s 1}$ is present.

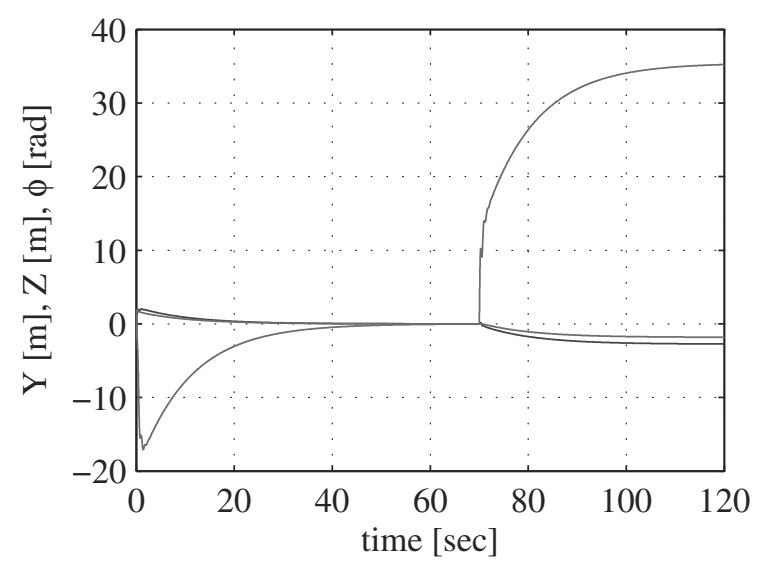

Fig. 5. PVTOL output when the fault $f_{a 3}$ is present.

PVTOL when the sensor fault $f_{s 1}$ occurs and Fig. 5] shows the case when the actuator fault $f_{a 3}$ occurs. As can be noticed in the case without failures, the states become the reference value and remain so, but when a fault occurs, the state deviates from the reference signal. Figure 6 shows
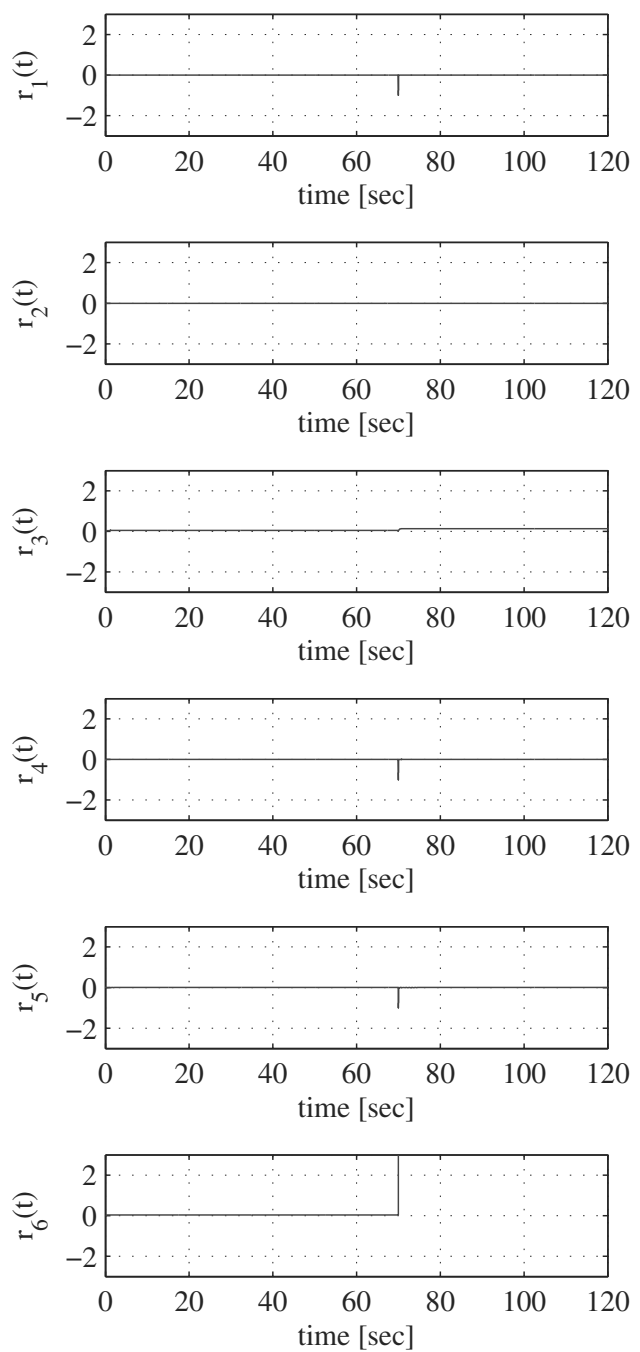

Fig. 6. Response of residuals to the sensor fault $f_{s 1}$.

the evolution of the residuals when the sensor fault $f_{s 1}$ occurs, where the residuals that differ from zero indicate sensitivity to the fault while the other residuals are zero at all time due their insensitivity to the fault. Note that the fault effect on the residuals 1,4 and 5 makes it difficult to make a decision about fault occurrence. Figure 7 shows the evolution of the residuals when the fault $f_{a 3}$ occurs, where the residuals $r_{3}$ and $r_{6}$ are different from zero due to their sensitivity to the fault while the others are insensitive to this fault.

\section{Conclusions}

In this work an approach to fault detection and isolation of nonlinear systems that admit a Hamiltonian representation 

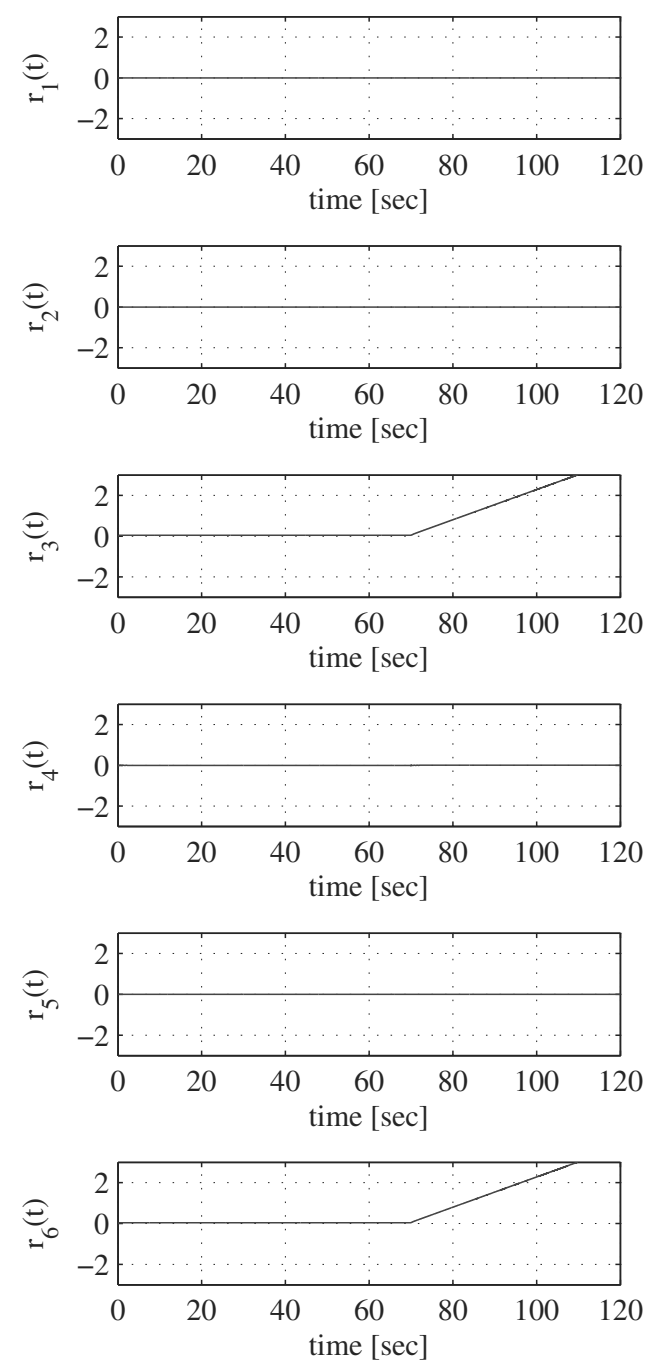

Fig. 7. Response of residuals to the actuator fault $f_{a 3}$.

was proposed. The isolation of faults is obtained by application of decoupling theory. Residual generation is proposed based on a novel variant of an observer for a system in a Hamiltonian representation. In particular, the case of a planar vertical take-off and landing aircraft model is analyzed. Starting from a Euler-Lagrange model representation, a Hamiltonian representation of the PVTOL is obtained. Faults were considered in all of the PVTOL sensors and control inputs. Decoupling is obtained from reasoning and algebraic manipulation. It was also possible to verify that subsystems sensitive to any fault maintain their generalized Hamiltonian representation which is present in PVTOL before performing the fault decoupling. This, in turn, simplifies the design of nonlinear observers as well as the fault diagnosis itself. The proposed approach was applied to model of PVTOL, with good results. All the faults considered were detected and isolated.

Future work includes a robustness study as well as physical implementation in a laboratory setup.

\section{Acknowledgment}

The authors wish to thank CONACyT-Mexico for the support of this work under the grant no. 178282.

\section{References}

Ailon, A. (2010). Simple tracking controllers for autonomous VTOL aircraft with bounded inputs, IEEE Transactions on Automatic Control 55(3): 737-743.

Al-Hiddabi, S., Shen, J. and McClamroch, N. (1999). A study of flight maneuvers for the PVTOL aircraft model, Proceedings of the American Control Conference, San Diego, CA, USA, Vol. 4, pp. 2727-2731.

Alcorta-Garcia, E. and Frank, P.M. (1997). Deterministic nonlinear observer-based approaches to fault diagnosis: A survey, Control Engineering Practice 5(5): 663-670.

Alcorta Garcia, E. (1999). Modelgestütze Residuengenerierung für die Diagnose von additiven and multiplicativen Fehler in dynamischen Systemen, Ph.D. thesis, Gerhard-Mercator University of Duisburg, Duisburg.

Apostol, T.M. (1967). Calculus I: One-variable Calculus, with an Introduction to Linear Algebra, 2nd Edn., Vol. 1, John Wiley \& Sons, New York, NY.

Bartyś, M. (2013). Generalized reasoning about faults based on the diagnostic matrix, International Journal of Applied Mathematics Computer Science 23(2): 407-417, DOI: 10.2478/amcs-2013-0031.

Blanke, M., Kinnaert, M. and Staroswiecki, J.L.M. (2006). Diagnosis and Fault-tolerant Control, 2nd Edn., Springer, Berlin.

Castillo, P., Dzul, A. and Lozano, R. (2004). Stabilization and tracking of a four-rotor mini rotorcraft, IEEE Transactions on Control System Technology 12(4): 510-517.

Castillo., P., Lozano, R. and Dzul, A. (2005). Modelling and Control of Mini-flying Machines, Springer, London.

Castillo, P., Lozano, R., Fantoni, I. and Dzul, A. (2002). Control design for the PVTOL aircraft with arbitrary bounds on the acceleration, Proceedings of the 41st IEEE Conference on Decision and Control, Las Vegas, NV, USA, Vol. 2, pp. 1717-1722.

Chen, J. and Patton, R.J. (1999). Robust Model Based Fault Diagnosis for Dynamic Systems, Kluwer Academic Publishers Group, Boston, MA.

DePersis, C. and Isidori, A. (2001). A geometric approach to nonlinear fault detection and isolation, IEEE Transactions on Automatic Control 46(6): 853-865.

Ding, S.X. (2008). Model-Based Fault Diagnosis Techniques, Springer, Berlin/Heidelberg. 
Ding, S.X. (2013). Model-Based Fault Diagnosis Techniques, 2nd Edn., Springer, London.

Do, K.D., Jiang, Z.P. and Pan, J. (2003). On global tracking control of a VTOL aircraft without velocity measurements, IEEE Transactions on Automatic Control 48(12): 2212-2217.

Etkin, B. and Reid, L. (1996). Dynamics of Flight, Stability and control, 3rd Edn., John Wiley and Sons, Hoboken, NJ.

Fantoni, I., Lozano, R. and Castillo, P. (2002). A simple stabilization algorithm for the PVTOL aircraft, 15th IFAC World Congress, Barcelona, Spain, pp. 1225-1225.

Frank, P.M. (1990). Fault diagnosis in dynamic systems using analytical and knowledge-based redundancy-a survey, Automatica 26(3): 459-474.

Frye, M., Ding, S., Qian, C. and Li, S. (2010). Fast convergent observer design for output feedback stabilisation of a planar vertical takeoff and landing aircraft, IET Control Theory and Applications 4(4): 690-700.

Isermann, R. (2006). Fault-Diagnosis Systems: An Introduction from Fault Detection to Fault Tolerance, 1st Edn., Springer, Berlin/Heidelberg.

Kościelny, J.M. and Łabęda-Grudziak, Z.M. (2013). Double fault distinguishability in linear systems, International Journal of Applied Mathematics and Computer Science 23(2): 395-406, DOI: 10.2478/amcs-2013-0030.

Krokavec, D. and Filasová, A. (2012). Novel fault detection criteria based on linear quadratic control performances, International Journal of Applied Mathematics and Computer Science 22(4): 929-938, DOI: 10.2478/v10006-012-0069-7.

Lin, F., Zhang, W. and Brandt, R. (1999). Robust hovering control of a PVTOL, IEEE Transactions on Control Systems Technology 7(3): 343-351.

Murray, R.M., Li, Z. and Sastry, S. (1994). A Mathematical Introduction to Robotic Manipulation, CRC Press, Boca Raton, FL.

Ortega, R., Loria, A., Nicklasson, P.J. and Sira Ramirez, H. (1998). Passivity-based Control of Euler Lagrange Systems: Mechanical, Electrical and Electromechanical Applications, Springer, London.

Rejon, V. and Aranda-Bricaire, E. (2006). Discrete-time stabilization of a PVTOL without roll angle and velocities measurement, Proceedings of the 45th IEEE Conference on Decision \& Control, San Diego, CA, USA, pp. 1521-1526.

Rodríguez Alfaro, L.H. (2014). Active Fault Tolerant Control of Hamiltonian Convergent Systems, Ph.D. thesis, Autonomous University of Nuevo Leon, San Nicolas de los Garza, (in Spanish).

Seliger, R. and Frank, P.M. (1991). Fault diagnosis by disturbance decoupled nonlinear observers, $C D C$ '91, Brighton, UK, pp. 2248-2253.

Sira Ramírez, H. and Cruz Hernández, C. (2001). Synchronization of chaotic systems: A generalized Hamiltonian systems approach, International Journal of Bifurcation and Chaos 11(5): 1381-1395.
Stevens, B.L. and Lewis, F.L. (2003). Aircraft Control and Simulation, John Wiley and Sons, Hoboken, NJ.

Thau, F.E. (1973). Observing the state of non-linear dynamic systems, International Journal of Control 17(3): 471-479.

van der Schaft, A. (2000). $L_{2}$ Gain and Passivity Techniques in Nonlinear Control, Lecture Notes in Control and Information Sciences, Vol. 218, Springer, Berlin/Heidelberg.

Vasiljevic, L.K. and Khalil, H.K. (2008). Error bounds in differentiation of noisy signals by high-gain observers, Systems \& Control Letters 57(10): 856-862.

Wood, R. and Cazzolato, B. (2007). An alternative nonlinear control law for the global stabilization of the PVTOL vehicle, IEEE Transactions on Automatic Control 52(7): 1282-1287.

Zavala, A., Fantoni, I. and Lozano, R. (2003). Global stabilization of a PVTOL aircraft model with bounded inputs, International Journal of Control 76(18): 1833-1844.

Zhang, Y. and Jiang., J. (2008). Bibliographical review on reconfigurable fault-tolerant control systems, Annual Reviews in Control 32(2): 229-252.

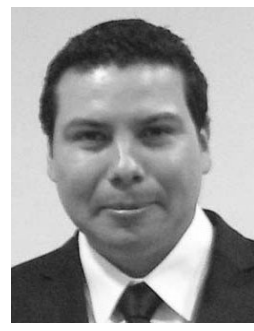

Luis H. Rodriguez-Alfaro was born in Villanueva, Zacatecas, Mexico, in 1985. He received the B.Sc. degree in electronics and communication engineering in 2008 from Universidad Autónoma de Zacatecas (UAZ), Mexico, the M.Sc. in electrical engineering (automatic control) in 2010 and the Dr.-Ing. in electrical engineering (automatic control) in 2014 from Universidad Autónoma de Nuevo León (UANL), Mexico. Since January 2014 he has been teaching at UANL. His areas of interest are fault diagnosis and fault tolerant control.

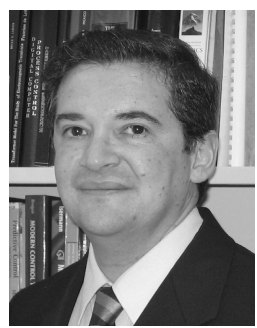

Efrain Alcorta-Garcia was born in Monterrey, Nuevo León, Mexico, in 1968. He received the B.Sc. degree in electronics and communication engineering and the M.Sc. in electrical engineering (automatic control) from Universidad Autónoma de Nuevo León (UANL), Mexico, in 1989 and 1992, respectively, and the Dr.-Ing. in electrical engineering (automatic control) from the Gerhard Mercator University of Duisburg, Germany, in 1999. Since 1999 he has held a teaching and research position at UANL. His research interests include model-based fault diagnosis, fault tolerant control and observers.

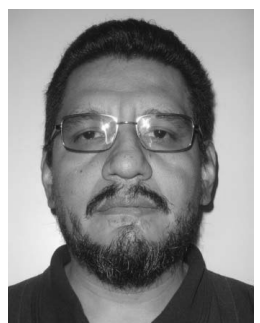

David Lara received the B.Sc. in electronic engineering from Madero Technology, Mexico, in 1996, the M.Sc. in automatic control and instrumentation from UAT, Mexico, in 2001, and the Ph.D. degree in TIS with a specialization in mechatronic control from UTC, France, in 2007. He has been a research professor at UAT, Campus Reynosa, since 2007. His research interests are in automatic control of mechatronic systems. 


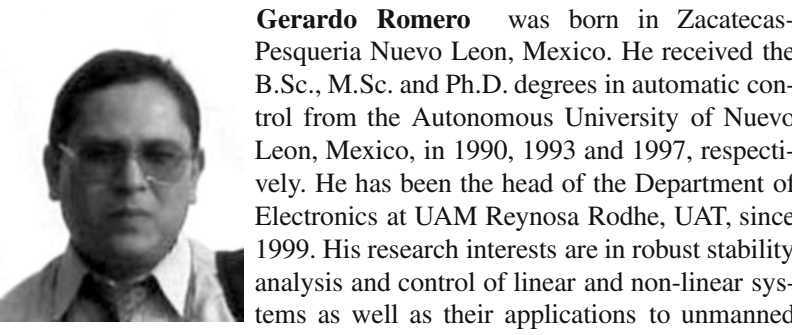
aerial vehicles and industrial processes.

Received: 28 January 2014

Revised: 15 September 2014 